\title{
NEURAL SLIDING MODE CONTROL FOR HYSTERESIS SYSTEMS
}

\author{
Chuntao Li, Yonghong Tan ${ }^{+}$ \\ Nanjing University of Aeronautics and Astronautics, 210016 Nanjing, China \\ +Guilin University of Electronic Technology, 541004 Guilin, China
}

\begin{abstract}
In this paper, a neural network based adaptive sliding mode control scheme for hysteretic systems is proposed. In this control scheme, a neural network model is utilized to describe the characteristic of hysteresis. Then, the adaptive neural sliding mode controller based on the proposed neural model is presented for a class of single-input nonlinear systems with unknown hysteresis. For the case where the output of hysteresis is unmeasurable, the neural network model is applied to estimate the effect of hysteresis. Based on the model-based estimation, the effect of hysteresis on the performance of the system is compensated. Copyright ( 12005 IFAC
\end{abstract}

Keywords: Neural networks; hysteresis; adaptive control; sliding-mode control, modelling.

\section{INTRODUCTION}

Usually hysteresis exists in piezoelectric actuators which are often used for micro-position devices in precision manufacturing engineering. However, hysteresis inherent in piezoelectric actuator severely constrains the performance of the system. It may result in undesirable accuracy or oscillations. The features of hysteresis are generally non-smooth and usually unknown. Thus it is a difficult task to remove its harmful effects by using traditional control strategies. Recently, Hwang et al (2001) used two neural networks to construct the inverse model of each branch of a single-loop hysteresis in order to implement the hybrid strategy consists of an intelligent variable-structure control and feedforward control. Tao and Kokotovic (1995) developed an adaptive inverse control scheme based on a simplified hysteresis model to cancel the effect of hysteresis. Ping Ge and Jouaneh (1996) developed a PID feedback control with an inverse static hysteresis model in the feedback loop. Selmic and Lewis (2001) proposed a back-stepping technique with an inverse backlash model for compensating the backlash nonlinearity, which is a special case of hysteresis. The drawback of the above-mentioned control schemes is that the direct inverse models have to be constructed to cancel the effects of hysteresis. However, in practice, the hysteresis hides in the plants or actuators so that it usually cannot be measured directly. Hence the control schemes depending on the inverse models in terms of the measured outputs of hysteresis will be very difficult for implementation. Generally, hysteresis contains more than one loops, the construction of the direct inverse model for multi-loop hysteresis will be very complex and difficult.

In order to compensate for the effect of hysteresis, a transform is developed to decompose the multi-valued mapping between the output and input of hysteresis into non-multivalued mapping. Then, a neural network is utilized to identify hysteresis based on the decomposition results. Under the assumption that plant states are all measurable, an adaptive sliding mode controller based on proposed neural model is proposed for the nonlinear plant preceded by unknown hysteresis.

\section{SYSTEMS WITH HYSTERSIS}

Consider a SISO nonlinear plant preceded by hystersis characteristic $H[\cdot]$ 


$$
\left\{\begin{array}{c}
\dot{x}_{1}=x_{2} \\
\dot{x}_{2}=x_{3} \\
\quad \vdots \\
\dot{x}_{n}=f(x, u) \\
u=H[v](t) \\
y=x_{1}
\end{array}\right.
$$

where $y(t)$ is the output; $x=\left[x_{1}, x_{2} \cdots x_{n}\right]^{T}$ is the state vector; $v(t)$ is the control input; $u(t)$ is the output of the actuator containing hysteresis which can be described by Preisach model (Polycarpou, 1996), and $f(x, u)$ is a smooth function that satisfies $\frac{\partial f}{\partial u} \neq 0$. The notation $H[v](t)$ denotes that the operator is dependent on the trajectory $v(t)$, which is not an instantaneous value.

The control objective is to design a control law for $v(t)$ to force the plant output $y(t)$ to follow a smooth prescribed trajectory $y_{d}(t)$. The desired states are defined as

$$
x_{d}(t)=\left[y_{d}, \dot{y}_{d}, \cdots y_{d}^{(n-1)}\right]^{T}
$$

with $y_{d}^{(n-1)}$ the $(n-1)^{\text {th }}$ derivative. The tracking errors are defined as

$$
e=\left[e_{1}, e_{2} \cdots e_{n}\right]^{T}=x-x_{d} .
$$

Assumption 1: $\left\|\left(x_{d}^{T}, y_{d}^{(n)}\right)\right\| \leq d$ where $\mathrm{d}>0$.

\section{MODELING HYSTERSIS}

Control of a system with hysteresis nonlinearity is difficult for its non-differentiable and multi-valued property. In order to describe hysteresis nonlinearity, various mathematical models have been proposed. Among them, Preisach model is the most popular model for the control design. This model can be described as

$$
u(t)=H[v](t)=\iint_{(\alpha, \beta) \in S} \mu(\alpha, \beta) \gamma_{\alpha, \beta}[v](t) d \alpha d \beta
$$

where $\gamma_{\alpha, \beta}[v](t)$ is the hystersis operator with $\alpha$ and $\beta$ corresponding to up and down switching values respectively. The operators are either +1 or -1 . $\mu(\cdot, \cdot): S \mapsto R$ is the weight function; $S=\left\{(\alpha, \beta) \mid v_{\min }(t) \leq \beta \leq \alpha \leq v_{\max }(t)\right\}$ is the triangle region, which can be subdivided into two time-varying sets, i.e.

$$
S^{+}:=\left\{(\alpha, \beta) \in S \mid \gamma_{\alpha, \beta}[v](t)=+1\right\} ; S^{-}:=\left\{(\alpha, \beta) \in S \mid \gamma_{\alpha, \beta}[v](t)=-1\right\} .
$$

$v_{\min }(t)$ and $v_{\max }(t)$ are the minimal and maximal value of $\mathrm{v}(\mathrm{t})$ for $t \in(-\infty, t]$. Therefore, $v_{\min }(t)$ and $v_{\max }(t)$ are varied as time $t$ changes.

Using the definition of $S^{+}$and $S^{-}$, (4) can be written as

$$
H[v](t)=\iint_{S^{+}} \mu(\alpha, \beta) d \alpha d \beta-\iint_{S^{-}} \mu(\alpha, \beta) d \alpha d \beta .
$$

From (5), one can see that Preisach model depends only on the interface $B[v](t)$ between $S^{+}$and $S^{-}$. Therefore, a mapping $\Psi$ is introduced to express the correspondence between Preisach model and $B[v](t)$

$$
H[v](t)=\Psi(B[v](t))
$$

Definition 1: For the given input $v(t)$, two boundaries $B[v]\left(t_{1}\right)$ and $B[v]\left(t_{2}\right)$ formed respectively at time $t_{1}$ and $t_{2}$ are called equal if the dominant extrema and current value $v\left(t_{1}\right)$, which forms the interface $B[v]\left(t_{1}\right)$, is equal to those of $B[v]\left(t_{2}\right)$. One describes the relationship between two boundaries in simple form as

$$
B[v]\left(t_{1}\right)=B[v]\left(t_{2}\right) .
$$

Remark 1: The dominant extrema are defined as the extrema of input $v(t)$, which consist of the integral boundary $B[v](t)$.

For $v(t)$ decreases or increases monotonically in $[t, t+\Delta t](\Delta t>0)$, (5) becomes

$$
\begin{aligned}
& H[v](t)=\left\{\begin{aligned}
H[v]\left(t_{e}\right)-2 \int_{\alpha_{e}}^{v(t)}\left[\int_{\alpha_{e}}^{\beta} \mu(\alpha, \beta) d \alpha\right] d \beta & \dot{v}(t) \leq 0 \\
H[v]\left(t_{e}\right)+2 \int_{\beta_{e}}^{v(t)}\left[\int_{\beta_{e}}^{\alpha} \mu(\alpha, \beta) d \beta\right] d \alpha & \dot{v}(t)>0
\end{aligned}\right. \\
& H[v]\left(t_{e}\right)=\iint_{S_{t e}^{+}} \mu(\alpha, \beta) d \alpha d \beta-\iint_{S_{t e}^{-}} \mu(\alpha, \beta) d \alpha d \beta
\end{aligned}
$$

where $\dot{v}>0$ or $\dot{v} \leq 0$ is just to show $\mathrm{v}(\mathrm{t})$ is monotonic signal in $[t, t+\Delta t] \cdot t_{e}$ is the time at which $\alpha_{e}=v\left(t_{e}\right)$ or $\beta_{e}=v\left(t_{e}\right)$ is the past dominant extremum adjacent to $v(t) \cdot S_{t e}^{+}$and $S_{t e}^{-}$are the integral area at time $t_{e}$, which keeps unchanged for $t \in[t, t+\Delta t]$. From (8), one obtains the derivative

$$
\frac{d H[v]}{d v}=\left\{\begin{array}{ll}
-2 \int_{\alpha_{e}}^{v(t)} \mu(\alpha, v(t)) d \alpha & \dot{v}(t) \leq 0 \\
+2 \int_{\beta_{e}}^{v(t)} \mu(v(t), \beta) d \beta & \dot{v}(t)>0
\end{array} .\right.
$$

From what is stated above, it is known that the relationship between the input and output of Preisach model is a multi-valued mapping. Hence it is quite difficult to utilize the conventional technique to determine $\mu(\alpha, \beta)$ in Preisach model since the conventional methods of system identification cannot handle the case of the systems with multi-valued mappings. However, we know that hysteresis described by Preisach model depends on its integral boundary $\mathrm{B}[\mathrm{v}](\mathrm{t})$. So that, we will give assumption 2 and lemma 1 shown in the following to set up a relationship between the output of hysteresis and the coordinate that represents the integral boundary.

Assumption 2: Let $v(t) \in C\left(R^{+}\right)$, where $R^{+}=\{t \mid t \geq 0\}$, and $C\left(R^{+}\right)$is the set of continuous functions on $\mathrm{R}^{+}$. Define a transform operator $\hat{h}[v](t)$ generated by Preisach model in a special case with a positive, bounded and piecewise continuous weight function $\hat{\mu}(\alpha, \beta)$, if there exist $t_{1}$ and $t_{2}\left(t_{1} \neq t_{2}\right)$, such that $v\left(t_{1}\right)=v\left(t_{2}\right), \hat{h}[v]\left(t_{1}\right)=\hat{h}[v]\left(t_{2}\right)$, and $\operatorname{sgn}\left(\left.\dot{v}\right|_{t_{1}}\right)=\operatorname{sgn}\left(\left.\dot{v}\right|_{t_{2}}\right)$ but $B[v]\left(t_{1}\right) \neq B[v]\left(t_{2}\right)$, the most adjacent and dominant extremum of $v\left(t_{1}\right)$ and that of $v\left(t_{2}\right)$ are not equal. From (10), the assumption is equivalent to:

$$
\left.\frac{d \hat{h}[v]}{d v}\right|_{t=t_{1}} \neq\left.\frac{d \hat{h}[v]}{d v}\right|_{t=t_{2}} .
$$

Lemma 1: For a given input $v(t)$ and the transform operator $\hat{h}[\cdot]$ satisfied assumption 2, then there exists an invertible mapping $\Gamma$ such that 


$$
\left(v(t), \hat{h}[v](t),\left.\operatorname{sgn}(\dot{v}) \frac{d \hat{h}[v]}{d v}\right|_{t}\right)=\Gamma(B[v](t)) .
$$

Theorem 1: For any hysteresis $H[\cdot]$ described by Preisach model, there exists a mapping $\mathbb{F}: R^{3} \mapsto R$ such that

$$
H[v](t)=\mathbb{F}\left(v(t), \hat{h}[v](t),\left.\operatorname{sgn}(\dot{v}) \frac{d \hat{h}[v]}{d v}\right|_{t}\right)
$$

where $v(t)$ and $\hat{h}[v](t)$ satisfy the assumption 2 .

The proof of Lemma 1 and theorem 1 are omitted. Please refer to (Li and Tan, 2004).

\section{SLIDING MODE CONTROL}

Define a scalar function as follows to describe the dynamics of tracking error

$$
s(t)=\left(\frac{d}{d t}+\lambda\right)^{n-1} e_{1}(t)=\left[\Lambda^{T}, 1\right] e(t)
$$

with $\lambda$ is a positive constant and $\Lambda=\left[\lambda^{n-1},(n-1) \lambda^{n-2}, \frac{(n-1)(n-2)}{2} \lambda^{n-3} \cdots,(n-1) \lambda\right]^{T}$. The sliding surface $s(t)=0$ represents a linear differential equation whose solution implies that $e(t)$ converges to zero with time constant $\frac{n-1}{\lambda}$ (Wei and Sun 2000).

Differentiating (14) with respect to time and using (1) and(3), we obtain

$$
\dot{s}(t)=f(x, u)-y_{d}^{(n)}+\left[0, \Lambda^{T}\right] e(t) \cdot
$$

Since the function $f(x, u)$ and hysteresis nonlinearity may not be known, an invertible function $\hat{f}(x, v)$ is introduced. By adding and subtracting $\hat{f}(x, v)$ to (15), the system can be expressed as

$$
\dot{s}(t)=\delta+\tilde{f}(x, u, v)+\left[0, \Lambda^{T}\right] e(t)-y_{d}^{(n)}
$$

where $\delta=\hat{f}(x, v)$ is the pseudo-control input; $v=\hat{f}^{-1}(x, \delta) \quad ; \quad$ and $\quad \tilde{f}(x, u, v)=f(x, u)-\hat{f}(x, v)$. The function $\hat{f}(x, u)$, which is invertible with respect to its second argument $u$, represents any available approximation of $f(x, u)$. It may be constructed from approximately linear models. Additional requirements on $\hat{f}(x, u)$ will be specified latter.

For the modeling of hysteresis $u=H[v]$, some difficulties may be encountered due to its output is usually unmeasurable directly and its properties of multivalued mapping. In order to handle the problem of hysteresis' output is unmeasurable directly, in this paper, we propose a method to estimate the effect of hysteresis through the residual $\tilde{f}(x, u, v)$. Therefore, we apply neural networks to implement the approximation of $\tilde{f}(x, u, v)$. A three-layer neural network, which is consisted of $n_{2}$-hidden neurons and $\mathrm{n}_{3}$-output neurons with linear output activation functions, can be described in term of vectors as

$$
y=W^{T} \sigma\left(V^{T} x_{n n}\right)
$$

where $\quad V=\left[V_{i, j}\right] \quad i=1,2, \cdots n_{1} \quad, \quad j=0,1 \cdots n_{2} \quad$; and $W=\left[W_{j, k}\right] \quad k=1,2 \cdots n_{3}$; are the interconnecting weight matrices, $\sigma(\cdot)$ is the sigmoidal function.

In order to tackle the problem of multivalued mapping in the procedure of using neural networks for hysteresis modeling, in terms of theorem 1 , we obtain that $\tilde{f}(x, u, v)=\tilde{f}\left(x, v, \mathbb{F}\left(v, \hat{h}[v], \operatorname{sgn}(\dot{v}) \frac{d \hat{h}}{d v}\right)\right)$.

Lemma 2: For a given $\varepsilon_{N}>0$, there exists a set of bounded weights $W$ and $V$, such that model error $\tilde{f}\left(x, v, \mathbb{F}\left(v, \hat{h}[v], \operatorname{sgn}(\dot{v}) \frac{d \hat{h}}{d v}\right)\right)$ can be approximated over a compact set $\Omega \subset R^{n+2}$ by a three-layer feedforward neural network

$$
\begin{aligned}
& \tilde{f}\left(x, \mathbb{F}\left(v, \hat{h}[v], \operatorname{sgn}(\dot{v}) \frac{d \hat{h}}{d v}\right), v\right)=W^{T} \sigma\left(V^{T} x_{n n}\right)+\varepsilon\left(x_{n n}\right), \\
& \left\|\varepsilon\left(x_{n n}\right)\right\| \leq \varepsilon_{N},
\end{aligned}
$$

where

$x_{n n}=\left[1, v(t), \cdots, v(t-m d), \hat{h}[v](t), \cdots, \hat{h}[v](t-m d), x_{d}, e\right]^{T} ; \quad v$ and $\hat{h}[\cdot]$ satisfy assumption 2. $d>0$ is the time delay; $m \geq 1, \varepsilon\left(x_{n n}\right)$ is the approximation error; $w$ and $v$ are the optimal weights that minimize $\varepsilon\left(x_{n n}\right)$ for all $x_{n n} \in \bar{\Omega} \subset R^{2(n+m)+3}$. The proof of lemma 2 is omitted (Barron, 1993; Calise et al, 2001).

Remark 2: Lemma 2 shows that neural network can directly be applied to estimate the influence of hysteresis which hidden in the residual $\tilde{f}(x, u, v)$. Based on Lemma 2, one is able to construct a compensator to eliminate the hysteresis influence without relying on the inverse model and measuring output of hytsteresis.

Assumption 3: The optimal weights $W$ and $V$ are respectively bounded by $W_{p}$ and $V_{p}$, i.e. $\|W\|_{F} \leq W_{p}$ and $\|V\|_{F} \leq V_{p}$, with $W_{p}$ and $V_{p}$ are known positive values, where \|\|$_{F}$ represents Frobenius norm.

Define the pseudo-control as

$$
\delta=y_{d}^{(n)}-K s(t)-\left[0, \Lambda^{T}\right] e(t)-\delta_{a d}+\delta_{r}
$$

where $\delta_{r}$ is a robust term selected for disturbance rejection; $K$ is a positive gain; and $\delta_{a d}$ is the output of the neural network used to approximate the model residual $\tilde{f}$, i.e.

$$
\delta_{a d}=\hat{W}^{T} \sigma\left(\hat{V}^{T} x_{n n}\right)
$$

where $\hat{W}$ and $\hat{V}$ are the estimates of the optimal weights $W$ and $V$. The controller architecture is shown in Fig. 4. From (16) and (19), $\tilde{f}(x, u, v)$ depends on $\delta_{a d}$ through $u, v$. However, $\delta_{a d}$ is designed to cancel the effect of $\tilde{f}(x, u, v)$. This requires the map $\delta_{a d} \mapsto \tilde{f}$ is a contraction, which holds under conditions: $\operatorname{sgn} f_{v}=\operatorname{sgn} \hat{f}_{v}$ and $0<\frac{1}{2}\left|f_{v}\right|<\left|\hat{f}_{v}\right| \quad$ (Hovakimyan et al, 2002; Calise et al, 2001) with $f_{v}=\frac{f(x, u)}{\partial u} \frac{\partial u}{\partial v}, \hat{f}_{v}=\frac{\hat{f}(x, v)}{\partial v}$. With(18)-(20), (16) can be rewritten as

$$
\dot{s}(t)=-K s(t)+W^{T} \sigma\left(V^{T} x_{n n}\right)-\hat{W}^{T} \sigma\left(\hat{V}^{T} x_{n n}\right)+\delta_{r}+\varepsilon .
$$

Define the weight estimation errors as

$$
\tilde{V}=V-\hat{V}, \tilde{W}=W-\hat{W}
$$

The Taylor series expansion of $\sigma\left(V^{T} x_{n n}\right)$ for a given $x_{n n}$ can be written as

$$
\sigma\left(V^{T} x_{n n}\right)=\sigma\left(\hat{V}^{T} x_{n n}\right)+\sigma^{\prime}\left(\hat{V}^{T} x_{n n}\right) \tilde{V}^{T} x_{n n}+o\left(\tilde{V}^{T} x_{n n}\right)^{2}
$$

where $\sigma^{\prime}\left(\hat{V}^{T} x_{n n}\right)$ is the Jacobian of $\sigma\left(\hat{V}_{n n}\right)$, and $o\left(\tilde{V}^{T} x_{n n}\right)^{2}$ denoting terms of second-order residue. 
Using (22), (23) and the following procedure (Wang et. al., 2001), the error dynamics of closed-loop can be expressed as

$$
\dot{s}(t)=-K s(t)+\left[\begin{array}{l}
\tilde{W}^{T}\left(\hat{\sigma}-\hat{\sigma}^{\prime} \hat{V}^{T} x_{n n}\right)+\hat{W}^{T} \hat{\sigma}^{\prime} \tilde{V}^{T} x_{n n} \\
+\delta_{r}+\omega+\varepsilon
\end{array}\right]
$$

where $\sigma=\sigma\left(V^{T} x_{n n}\right), \hat{\sigma}=\sigma\left(\hat{V}^{T} x_{n n}\right), \hat{\sigma}^{\prime}=\sigma^{\prime}\left(\hat{V}^{T} x_{n n}\right)$, and $\omega(t)$ is the disturbance term which is given by

$$
\omega(t)=W^{T} \sigma-\hat{W}^{T} \hat{\sigma}-\tilde{W}^{T}\left(\hat{\sigma}-\hat{\sigma}^{\prime} \hat{V}^{T} x_{n n}\right)-\hat{W}^{T} \hat{\sigma}^{\prime} \tilde{V}^{T} x_{n n} .
$$

It can also be written by

$$
\omega(t)=W^{T}(\sigma-\hat{\sigma})+W^{T} \hat{\sigma}^{\prime} \hat{V}^{T} x_{n n}-\hat{W}^{T} \hat{\sigma}^{\prime} V^{T} x_{n n} .
$$

The corresponding upper bound on the norm of $\omega(t)$ will be

$$
\begin{aligned}
& \|\omega(t)\| \leq\|V\|_{F}\left\|x_{n n} \hat{W}^{T} \hat{\sigma}^{\prime}\right\|_{F}+\|W\|\left\|\hat{\sigma}^{\prime} \hat{V}^{T} x_{n n}\right\|+\|W\|_{1} \\
& \|\omega(t)\| \leq \rho_{\omega} \phi_{\omega}\left(\hat{W}, \hat{V}, x_{n n}\right)
\end{aligned}
$$

where

$$
\begin{aligned}
& \phi_{\omega}=\left(\left\|x_{n n} \hat{W}^{T} \hat{\sigma}^{\prime}\right\|_{F}+\left\|\hat{\sigma}^{\prime} \hat{V}^{T} x_{n n}\right\|+1\right), \\
& \rho_{\omega}=\max \left(\left\|V_{F}\right\|,\|W\|,\|W\|_{1}\right) .
\end{aligned}
$$

In order to driving the adaptive law, a tuning error, $s_{\Delta}$, is introduced as follows

$$
\begin{aligned}
& s_{\Delta}=s-\Delta \text { sat }\left(\frac{s}{\Delta}\right), \\
& \operatorname{sat}\left(\frac{s}{\Delta}\right)= \begin{cases}1 & s>\Delta \\
\frac{s}{\Delta} & \|s\| \leq \Delta \\
-1 & s<-\Delta\end{cases}
\end{aligned}
$$

where $\Delta$ is an arbitrary positive constant. Hence we derive the following theorem:

Theorem 2: Suppose that assumptions 1, 2, and 3 are held. Consider closed-loop system (1), the sliding mode control law and the corresponding adaptive law are given by

$$
\begin{aligned}
& v(t)=\hat{f}^{-1}(x, \delta) \\
& \delta=y_{d}^{(n)}-K s(t)-\hat{W}^{T} \sigma\left(\hat{V}^{T} x_{n n}\right)-\left[0, \Lambda^{T}\right] e+\delta_{r} \\
& \delta_{r}= \begin{cases}-\hat{\varphi}\left(\phi_{\omega}+1\right) \frac{s_{\Delta}}{\left\|s_{\Delta}\right\|}+K \Delta s a t\left(\frac{s}{\Delta}\right) & \left\|s_{\Delta}\right\| \neq 0 \\
0 & \left\|s_{\Delta}\right\|=0\end{cases} \\
& \dot{\hat{W}}=F\left(\hat{\sigma}-\hat{\sigma}^{\prime} \hat{V}^{T} x_{n n}\right) s_{\Delta} \\
& \dot{\hat{V}}=R\left(x_{n n} s_{\Delta} \hat{W}^{T} \hat{\sigma}^{\prime}\right) \\
& \dot{\hat{\varphi}}=\gamma\left\|s_{\Delta}\right\|\left(\phi_{w}+1\right)
\end{aligned}
$$

where $\varphi=\max \left(\rho_{\omega}, \varepsilon_{N}\right), \hat{\varphi}$ is the estimate of $\varphi, \tilde{\varphi}=\varphi-\hat{\varphi}$, $F^{T}=F>0, R^{T}=R>0$, and $\gamma>0$. If the initial error $(e(0), \tilde{W}(0), \tilde{V}(0)) \in \Omega_{r}$ (ref. to the definition shown in (44)) the signals $e, \hat{W}, \hat{V}$ and $\hat{\varphi}$ are all ultimately bounded. Proof: Consider the candidate of Lyapunov function as

$$
L=\frac{1}{2} s_{\Delta}^{2}+\frac{1}{2} \operatorname{tr}\left(\tilde{W}^{T} F^{-1} \tilde{W}\right)+\frac{1}{2} \operatorname{tr}\left(\tilde{V}^{T} R^{-1} \tilde{V}\right)+\frac{1}{2} \tilde{\varphi}^{T} \gamma^{-1} \tilde{\varphi} \cdot
$$

The derivative of $\dot{L}$ with respect to time is given by

$$
\dot{L}=s_{\Delta} \dot{s}_{\Delta}+\operatorname{tr}\left(\tilde{W}^{T} F^{-1} \dot{\tilde{W}}\right)+\operatorname{tr}\left(\tilde{V}^{T} R^{-1} \dot{\tilde{V}}\right)+\tilde{\varphi}^{T} \gamma^{-1} \dot{\tilde{\varphi}}
$$

where $\dot{s}_{\Delta}=0$ for $\|s\| \leq \Delta$ and $\dot{s}_{\Delta}=\dot{s}$ for $\|s\|>\Delta$. According to the adaptive law (34)-(36), $\dot{L}=0$ for $\|s\| \leq \Delta$. Therefore, the remaining of this proof deals strictly with the case of $\|s\|>\Delta$. Using (24), it yields

$$
\begin{aligned}
& \dot{L}=-K s_{\Delta} s+\operatorname{tr} \tilde{W}^{T}\left[F^{-1} \dot{\tilde{W}}+\left(\hat{\sigma}-\hat{\sigma}^{\prime} \hat{V}^{T} x_{n n}\right) s_{\Delta}\right] \\
& +\operatorname{tr} \tilde{V}^{T}\left(R^{-1} \dot{\tilde{V}}+x_{n n} s_{\Delta} \hat{W}^{T} \hat{\sigma}^{\prime}\right)+s_{\Delta}\left(\delta_{r}+\omega+\varepsilon\right)+\tilde{\varphi}^{T} \gamma^{-1} \dot{\tilde{\varphi}}
\end{aligned}
$$

Based on (34), (35) and the fact $\dot{\tilde{W}}=-\dot{\hat{W}}, \dot{\tilde{V}}=-\dot{\hat{V}}$, Equ. (39) can be rewritten as

$$
\dot{L}=-K s_{\Delta} s+s_{\Delta}\left(\delta_{r}+\omega+\varepsilon\right)+\tilde{\varphi}^{T} \gamma^{-1} \dot{\tilde{\varphi}} .
$$

In terms of (18), (28) and $\varphi=\max \left(\rho_{\omega}, \varepsilon_{N}\right)$, we obtain

$$
\dot{L} \leq-K s_{\Delta} s+s_{\Delta} \delta_{r}+\left\|s_{\Delta}\right\| \varphi\left(\phi_{\omega}+1\right)+\tilde{\varphi}^{T} \gamma^{-1} \dot{\tilde{\varphi}} .
$$

Using (36) and $s_{s=s_{\Delta}}+\Delta \operatorname{sat}\left(\frac{s}{\Delta}\right)$, (41) becomes

$$
\dot{L} \leq-K s_{\Delta}^{2}-K \Delta s_{\Delta} \operatorname{sat}\left(\frac{s}{\Delta}\right)+\left\|s_{\Delta}\right\| \hat{\varphi}\left(\phi_{\omega}+1\right)+s_{\Delta} \delta_{r} .
$$

Using(33), (42) can be expressed as

$$
\dot{L} \leq-K s_{\Delta}^{2} \text {. }
$$

Since (18) holds on a compact set $\Omega$, all the states needed to be remained in this compact set for all $t>0$ in order for (43) to be valid. Introducing compact set $\Omega_{l}=\{(e, \tilde{W}, \tilde{V}) \mid L(t) \leq l, 0<l\}$, constant $l^{*}$ is determined by $l^{*}=\sup _{l \in R^{+}}\left\{l \mid \forall(e, \tilde{W}, \tilde{V}) \in \Omega_{l},(x, u, v) \in \Omega\right\}$. Define the compact set

$$
\Omega_{r}=\left\{(e, \tilde{W}, \tilde{V}) \mid L(t) \leq r \leq l^{*}\right\}
$$

Then for any $(e(0), \tilde{W}(0), \tilde{V}(0)) \in \Omega_{r}$ it follows from (37) and (43) that $\mathrm{L}(\mathrm{t})$ is bounded from above by $r$, which implies that $(x, u, v) \in \Omega$ for all $t \geq 0$.

According to (43), one can obtain that $s_{\Delta}, \tilde{W}, \tilde{V}, \tilde{\varphi} \in L_{\infty}$. Since $L(t)$ is bounded from below and is nonincreasing with time, it has a limit, i.e., $\lim _{t \rightarrow \infty} L(t)=L(\infty)$. Using (43) and the fact that $s_{\Delta}=0$ for $\|s(t)\| \leq \Delta$, we have

$$
\int_{0}^{\infty} s_{\Delta}^{2}(t) d t \leq \frac{L(0)-L(\infty)}{K}<\infty
$$

which implies that $s_{\Delta} \in L_{2}$. It has been shown that every term in right hand side of (21) is bounded, hence $\dot{s}_{\Delta}$ is also bounded, i.e., $\dot{s}_{\Delta} \in L_{\infty}$. From $s_{\Delta} \in L_{2}, \dot{s}_{\Delta} \in L_{\infty}$ and $s_{\Delta} \in L_{\infty}$, we have $\lim _{t \rightarrow \infty} s_{\Delta}(t)=0$. This implies that $s(t)$ converges to the region $\|s(t)\| \leq \Delta$. We can also establish that the tracking errors are bounded from above by $\left\|e_{i}\right\| \leq 2^{i-1} \lambda^{i-n} \Delta, i=1,2, \cdots n$ (Tao and Kokotovic, 1995). According to the assumption 1-3, one can derive that all the signal in the closed-loop system is ultimately bounded.

\section{SIMULATION}

Consider a nonlinear systems described by

$$
\left\{\begin{array}{l}
\dot{x}_{1}=x_{2} \\
\dot{x}_{2}=x_{1} x_{2}+\frac{u^{3}}{1+x_{1}^{2}+x_{2}^{2}} \\
u=H[v](t) \\
y=x_{1}
\end{array}\right.
$$

the hysteresis $H[\cdot]$ is generated by summation of $\mathrm{N}=160$ backlashes, which can be described as

$$
H[v](t)=\sum_{i=1}^{N} u_{i} ; \quad \dot{u}_{i}=\left\{\begin{array}{ll}
\dot{v}(t) & \dot{v}(t)>0, u_{i}(t)=v(t)-\frac{d_{i}}{2} \\
\dot{v}(t) & \dot{v}(t)<0, u_{i}(t)=v(t)+\frac{d_{i}}{2} \\
0 & \text { otherwise }
\end{array} .\right.
$$

where $d_{i}$ s are uniformly distributed in [0,4], and all backlash are initialized to zero. We also assume that $\mathrm{f}(\mathrm{x}, \mathrm{u})$ is unkown for neural controller. The output $y(t)$ 
is required to track a desired trajectory $y_{d}(t)=0.5 e^{-\frac{t}{5}} \sin 3 t+\sin t+1$.A three-layer feedforward neural network, which is consisted of $n_{1}=9$ input nerons, $\mathrm{n}_{2}=20$ hidden neurons with sigmoidal activation functions and $n_{3}=1$ output neuron, is used to approximate $\tilde{f}$. The neural network weight matrices $\hat{W}$ and $\hat{V}$ are set to zero as the initialization.

The other parameters are chosen as

$\lambda=2, \quad F=20 I, R=20 I, \quad \gamma=5, \quad K=2, \Delta=0.01$,

$\hat{f}(x, v)=v, \quad x(0)=[0.5,2.0]^{T}, \hat{\varphi}(0)=2, d=0.01$,

$x_{n n}=\left[1, y_{d}, \dot{y}_{d}, e_{1}, e_{2}, v(t), v(t-d), \hat{H}[v](t), \hat{H}[v](t-d)\right]^{T}$,

where $I$ is a suitable identity matrix;. The transform operator $\hat{h}[\cdot]$ used to extract the boundary is choosen as

$$
\hat{h}[v](t)=\iint_{S} \gamma_{\alpha, \beta}[v](t) d \alpha d \beta .
$$

where $\mu(\alpha, \beta)=1$, so that $\hat{h}[\cdot]$ meets the requirement of assumption 2. In $(-\infty, 0]$, we set $v_{\min }(0)=v_{\max }(0)=0$, which implies $\hat{h}[v](0)=0$. In order to compare the tracking performance of controller with and without compensation, a PID controller is designed for (46) in which influence of hysteresis nonlinearity is ignored. the control signal $\mathrm{v}(\mathrm{t})$ is designed as

$$
v=-K_{I} \int_{0}^{t} e_{1}(t) d t-K_{P} e_{1}(t)-K_{d} e_{2}(t)
$$

with $K_{I}=8, K_{P}=4, K_{d}=2$. The tracking performances of the closed-loop system are shown in Fig. 1. Figs. (1a) and (1b) respectively illustrate the tracking performance in the case of using the proposed controller with the neural model and using PID controller without hysteresis compensation. $\mathrm{x}_{1}$ here is, in fact, the system output. The performance of $\mathrm{x}_{1}$ reflects the behavior of the closed-loop system. Figs. (1c), on the other hand, showes the tracking errors in both cases of the control schemes with and without hysteresis compensation.

The control signals of both control strategies both plotted in Fig. 2. Clearly, we can see that the proposed control approach with neural network based hysteresis compemsator can remove the oscillation caused by hysteresis and improve the tracking accuracy of the system. The proposed control method has derived much better performance than the control scheme without hysteresis comensation. The hysteresis responses of close-loop system with both different control schemes are shown in Fig. 3.

\section{CONCLUSIONS}

In order to apply neural network to modeling the multivalued hysteresis nonlinearity, a transform is firstly defined to construct the non-multivalued mapping between the output of hysteresis and an introduced coordinate. Then, an adaptive sliding mode controller for a nonlinear plant preceded by a unknown hysteresis is developed. In the control scheme, a neural network based estimator is used to forecast the effect of hysteresis. Therefore, the controller can cancel effect of hysteresis on the plant. The advantage of the proposed control approach is that it will be more useful to the practical process in engineering where the output of hysteresis is usually unmeasurable.

\section{ACKNOWLEDGEMENT}

This research is partially supported by National Science Foundation of China (NSFC Grant No.: 50265001) and Guangxi Science Foundation (GXSF Grant No.: 0339068).

\section{REFERENCES}

Barron, A. (1993). Universal approximation bounds forsuperposition of a sigmoidal function. IEEE Trans. Inform. Theory, 39, 930-945.

Calise, A. J., H. Naira, I. Moshe (2001). Adaptive output feedback control of nonlinear systems using neural networks. Automatica, 37, 1201-1211.

Ge, P., M. Jouaneh (1996). Tracking Control of a Piezoceramic Actuator. IEEE Trans. Control Systems Technology, 4(3), 209-216.

Hovakimyan, N. et al (2002). Adaptive Output Feedback Control of Uncertain Nonlinear systems Using single-Hidden-Layer Neural Networks. IEEE Trans. Neural Networks, 13(6), 1420-1431.

Wang, C.-L., C. Jan, and Y.-H. Chen (2001) Piezomechanics Using Intelligent VariableStructure Control. IEEE Trans. Industrial Electronics, 48(1), 47-59.

Kim, B. S. and A. J. Calise (1997). Nonlinear Flight Control Usng Neural Netwirks. J. Guidance, Control and Dynamics, 20(1), 26-33.

Leitner, J., A. Calise and J. V. R. Prasad (1997). Analysis of Adaptive Neural Networks for Helicopter Flight control. J. Guidance, Control and dynamics, 20(5),.973-979.

Lewis, F., A. Yesildirek, K. Liu (1996) Multilayer Neural -Net Robot Controller with Guaranteed Tracking Performance. IEEE Trans. Neural Networks, 7(2), 388-399.

Li, Chuntao, Y. Tan (2004). A Neural Networks Model for Hysteresis Nonlinearity. Sensors \& Actuators: A. Physical, 112(1), 49-54.

Mayergoz, D., G. Friedman (1991). Mathematical models of hysteresis. Springer-Verlag, New York.

Polycarpou, M. (1996). Stable Adaptive Neural Control Scheme for Nonlinear Systems. IEEE Trans. Automactic Control, 41(3), 447-451.

Selmic, R., F. Lewis (2001). Neural Net Backlash Compensation with Hebbian Tuning Using Dynamic Inversion. Automatica 37, 1269-1277.

Slotine, J. E. and W. Li (1995). Applied Nonlinear Control. Prentice Hall, Englewood Cliffs, NJ.

Su, C.-Y., Y. Tan, Y. Stepanenko (2000). Adaptive Control of A Class of Nonlinear Systerms Preceded by an Unknown Backlash-like Hysteresis. Proc. $39^{\text {th }}$ IEEE Conf. Decsion and Control, Sydney, Australia, 1459-1464.

Tao, G., P. Kokotovic (1995). Adaptive Control of Plant with Unknown Hysteresis. IEEE Trans. Automatic Control, 40(2), 200-213.

Wei, J., C. Sun (2000). Construting Hysteresis Memory in Neural Networks. IEEE Trans. on 
Systems, Man and Cybernetics Part B: Cybernetics, 30(4), 601-608

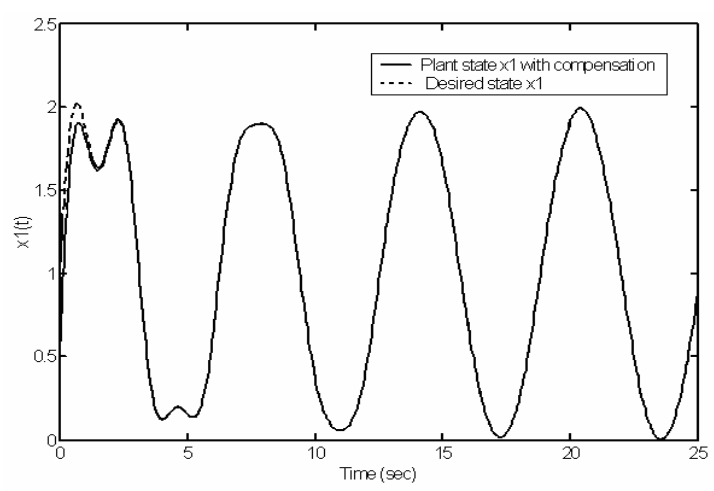

Fig.1a The performance of state $x_{1}$ with neural compensator

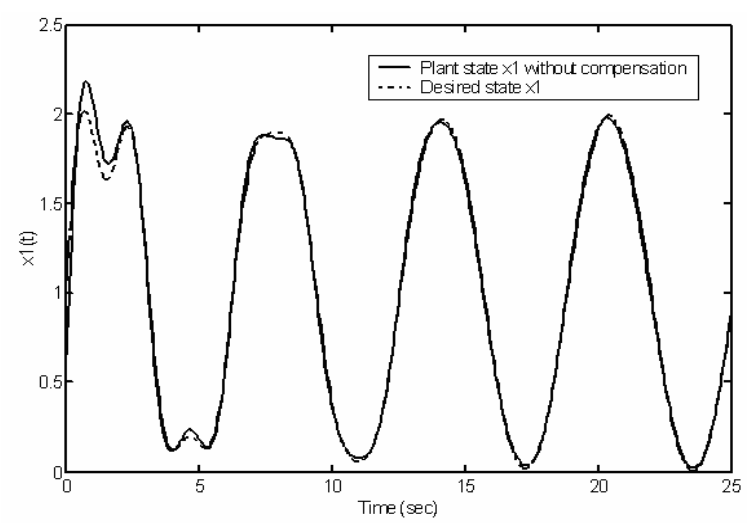

Fig.1b The performance of state $x_{1}$ without compensation

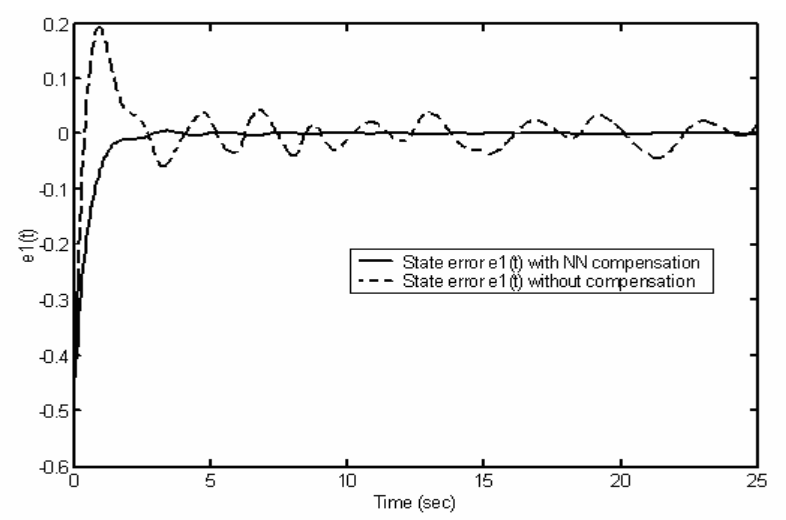

Fig. 1c The tracking eror of plant state $x_{1}$

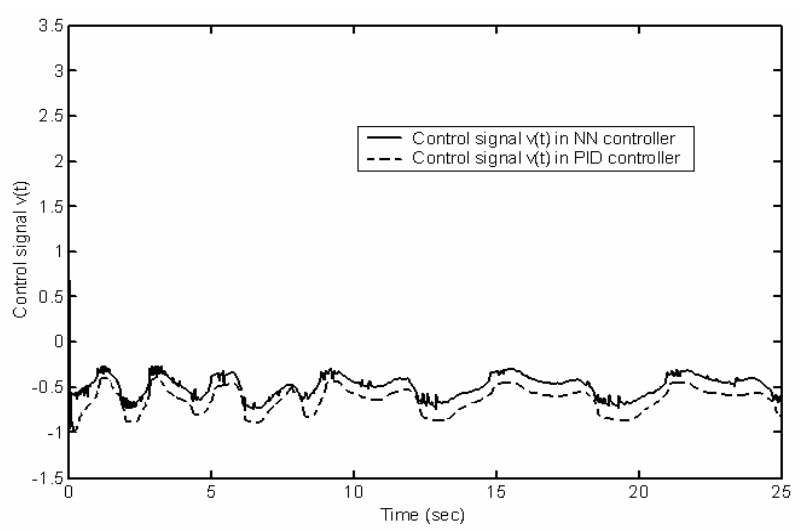

Fig.2. Control signal $v(\mathrm{t})$ in both $\mathrm{NN}$ and PID controllers

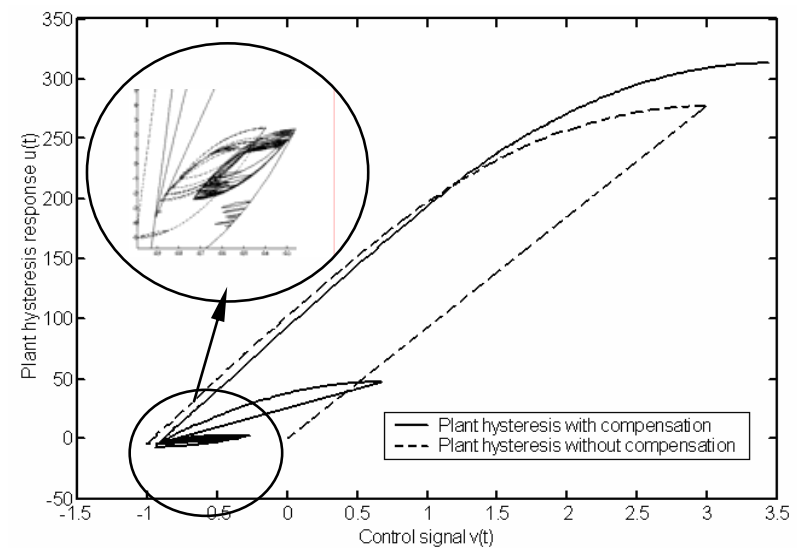

Fig.3 Hysteresis in closed-loop systems with and without compensation 